\section{Multimorbidade e uso de serviços de saúde em indivíduos com restrição de atividades habituais: Estudo Pró-Saúde}

\author{
Multimorbidity and use of health services by \\ individuals with restrictions on habitual \\ activities: the Pró-Saúde Study
}

\author{
Multimorbilidad y uso de servicios de salud \\ en individuos con restricción de actividades \\ habituales: Estudio Pro-Salud
}

\section{Resumo}

O objetivo do estudo foi avaliar o uso de serviços de saúde e sua associação com diferentes medidas de multimorbidade. Trata-se de um estudo transversal aninhado a uma investigação longitudinal de funcionários técnico-administrativos no Município do Rio de Janeiro, Brasil: Estudo Pró-Saúde. Foram analisados dados coletados na fase 2(2001-2002), sendo a população de estudo composta por 733 indivíduos que relataram restrição de atividades habituais por problemas de saúde nos 15 dias anteriores à coleta de dados. Busca por serviço de saúde (variável de desfecho) foi utilizada como proxy para uso de serviços de saúde. Multimorbidade foi avaliada por meio de contagem simples e de uma escala cumulativa (Cumulative Illness Rating Scale), gerando quatro variáveis de exposição: número de morbidades autorrelatadas, multimorbidade (2 ou mais morbidades), escore total e número de sistemas afetados. Nas análises estratificadas por sexo, foram utilizados modelos de regressão de Poisson com variância robusta ajustados por idade e escolaridade. Mulheres apresentaram em média valores mais altos para todas as medidas, e 51\% foram classificadas com multimorbidade. Ter multimorbidade aumentou em 43\% (IC95\%: 1,11-1,84) a probabilidade de utilizar os serviços de saúde em homens, enquanto para as mulheres não houve associação estatisticamente significativa. Para os homens, a cada morbidade adicional, a probabilidade de utilizar serviço de saúde aumentou em 14\% (IC95\%: 1,05-1,24). Diferenças no uso de serviços de saúde e multimorbidade segundo sexo são evidentes. Conhecer tais padrões torna-se relevante para a prestação de um cuidado eficiente, coordenado e seguro para pessoas com multimorbidade.

Múltiplas Morbidades; Comorbidade; Acesso aos Serviços de Saúde; Doença Crônica; Sistemas de Saúde
Ana Sara Semeão de Souza 1

Eduardo Faerstein 1

Guilherme Loureiro Werneck 1

\author{
Correspondência \\ A. S. S. Souza \\ Instituto de Medicina Social, Universidade do Estado do \\ Rio de Janeiro. \\ Rua São Francisco Xavier 524, Bloco E, 7o andar, \\ Rio de Janeiro, RJ 20550-013, Brasil. \\ anasarasemeao@gmail.com \\ 1 Instituto de Medicina Social, Universidade do Estado do Rio de \\ Janeiro, Rio de Janeiro, Brasil.
}




\section{Introdução}

O progressivo processo de envelhecimento da população, a crescente exposição a fatores ambientais, mudanças de estilos de vida e progressos na efetividade dos cuidados de saúde levaram a um aumento na prevalência de doenças crônicas não transmissíveis (DCNT) globalmente 1,2,3. No Brasil, assiste-se a processo similar, com deslocamento da carga de morbimortalidade dos grupos mais jovens aos mais idosos e advento de um perfil epidemiológico em que a morbidade por DCNT se tornou dominante 4.

Nesse contexto, a multimorbidade, definida como a ocorrência de duas ou mais doenças em um mesmo indivíduo, tem sido objeto de crescente interesse 5,6,7,8,9,10,11. Considerando a sua prevalência, gravidade, impacto na qualidade de vida e a possibilidade de manejo, a multimorbidade tem se tornado uma prioridade para a saúde pública ${ }^{12}$. No Brasil, estudo recente apontou que a multimorbidade é mais comum em indivíduos com menor escolaridade e sua prevalência aumenta com a idade 5 . Observou-se também que, em geral, ocorre em idades mais jovens do que em países mais ricos e 10 anos mais precocemente em mulheres em comparação aos homens 5 .

Taxas mais elevadas de DCNT contribuem para altos níveis de absenteísmo, presenteísmo e aposentadoria antecipada. O presenteísmo é caracterizado pela presença do funcionário no trabalho, porém sem desempenhar plenamente suas atividades laborais por causa de problemas físicos ou mentais 13,14. Para o Brasil, em 2015, estimou-se em 5,4\% (US\$ 129,8 bilhões) do total do Produto Interno Bruto (PIB) o custo econômico devido a mortes por DCNT e perdas de produtividade pelo absenteísmo e presenteísmo 15 . Um perfil de saúde da população no qual as doenças crônicas e suas complicações são mais prevalentes resulta em mudanças no padrão de utilização dos serviços de saúde e no aumento de gastos, considerando a necessidade de incorporação tecnológica para seu tratamento 4 .

Assim, admite-se que pessoas com várias doenças têm elevadas taxas de utilização de serviços de saúde, o que tem sido confirmado por alguns estudos 16,17,18. Entretanto, o onus da multimorbidade tem sido tipicamente estudado em países de alta renda, onde a sua prevalência e determinantes socioeconômicos, assim como o seu impacto sobre a utilização de serviços de saúde, estão mais bem estabelecidos 19,20,21. Além disso, há informações limitadas sobre os padrões de utilização de cuidados de saúde relacionada com várias doenças nos países em desenvolvimento 22 .

O presente estudo teve por objetivo avaliar o uso de serviços de saúde e a sua associação com diferentes medidas de multimorbidade.

\section{Métodos}

\section{População de estudo}

Trata-se de um estudo transversal aninhado em uma investigação longitudinal de funcionários técnico-administrativos de uma universidade localizada no Município do Rio de Janeiro, tendo como foco principal os determinantes sociais da saúde - Estudo Pró-Saúde. Até o momento foram conduzidas quatro fases $(1999,2001,2006$ e 2012), sendo a linha de base constituída pelos indivíduos elegíveis que participaram simultaneamente nas duas primeiras fases (3.253 participantes, $78,2 \%$ dos elegíveis) 23 . A população de estudo foi composta por participantes que referiram restrição das atividades habituais por motivo de saúde nas duas semanas anteriores à pesquisa, de acordo com a seguinte pergunta: "Nas últimas duas semanas, você ficou impedido(a) de realizar alguma de suas atividades habituais (por exemplo, trabalho, estudo, lazer ou tarefas domésticas) por algum problema de saúde que você teve ou tem?".

A população de estudo se restringiu a esses indivíduos pelo fato de a pergunta de uso de serviço de saúde estar atrelada à pergunta referida acima. Relataram ter impedimento de realizar suas atividades habituais 738 indivíduos. Foram excluídos aqueles que não haviam respondido à pergunta sobre uso de serviços de saúde (4 participantes) e com idade superior a 69 anos (1 participante). A população de estudo foi então composta por 733 indivíduos. 


\section{Variáveis de estudo}

Os dados foram coletados por meio de questionários autopreenchíveis 24. Busca por assistência ou atendimento em hospital e consultório médico (particular ou plano de saúde) foi utilizada como proxy do uso de serviços de saúde, sendo avaliado de forma dicotômica (sim/não). A pergunta referente ao uso de serviço de saúde foi: "Agora, pense no principal problema de saúde que você mencionou na pergunta anterior. Você procurou algum tipo de assistência ou atendimento para tratar desse problema?”.

Busca por serviços de saúde, apesar de não representar a totalidade da dimensão do uso de serviços, foi escolhida como proxy por conta dos elevados índices de demandas atendidas observados em alguns inquéritos nacionais, sugerindo que essa variável apresente boa representatividade da variável de origem $25,26,27,28$.

Multimorbidade foi avaliada por meio de contagem simples e de uma escala cumulativa (Cumulative Illness Rating Scale - CIRS).

$\mathrm{Na}$ contagem simples, foram utilizadas as seguintes informações autorrelatadas de condições diagnosticadas por médicos: hipertensão, dislipidemia, diabetes, infarto do miocárdio, angina, acidente vascular cerebral, asma, enfisema, cálculo vesicular, úlcera péptica, lesão por esforço repetitivo (LER), artrose, hérnia de disco, hipertireoidismo e hipotireoidismo. As seguintes morbidades foram agrupadas em uma única: infarto do miocárdio e angina (“doenças coronarianas”), asma e enfisema ("problemas pulmonares") e hiper e hipotireoidismo ("problemas da tireoide").

Todas as morbidades tiveram o mesmo peso (1) na contagem do total de morbidades. Pela contagem, foram construídas duas variáveis: uma contínua (soma total de morbidades) denominada "número de morbidades", e outra dicotômica ("multimorbidade") definida como a presença de duas ou mais morbidades (sim) e uma ou nenhuma morbidade (não).

A versão modificada da CIRS 29 é estruturada em 14 sistemas/órgãos que são pontuados segundo o grau de comprometimento do agravo. Cada sistema é ponderado de 0 a 4 pontos, e a pontuação de cada sistema deve seguir manual instrutivo e julgamento clínico do avaliador. Quando para o mesmo sistema houver morbidades com diferentes pontuações, prevalece a pontuação mais alta 29.

Para preenchimento da CIRS, foram utilizadas informações das duas primeiras fases do estudo (1999 e 2001) acerca de morbidades autorrelatadas, uso de medicamentos, história de tabagismo e etilismo, problemas de saúde (outros), informação sobre hospitalizações e cirurgias passadas. As principais morbidades incluídas na CIRS foram: hipertensão, dislipidemia, diabetes, infarto do miocárdio, angina, acidente vascular cerebral, asma, enfisema ou bronquite crônica, cálculo renal, cálculo vesicular, úlcera péptica, gastrite, LER, artrose, hérnia de disco, hipertireoidismo, hipotireoidismo e tuberculose. Diferentemente da contagem simples, a CIRS agrega, além das morbidades, condições que indicam gravidade dessas morbidades dentro de cada sistema/órgão.

A CIRS é uma escala de mensuração de morbidades que inclui em sua avaliação o nível de gravidade delas, classificadas dentro de sistemas ou órgãos. Com base na classificação de cada sistema ou órgão (0 a 4), a CIRS fornece cinco diferentes medidas: (1) o número de sistemas que receberam uma classificação > 1 (0 a 14); (2) a soma total das classificações atribuídas a cada sistema (escore total, 0 a 56); (3) um índice de gravidade, calculado pela razão entre o escore total e o número de sistemas afetados; (4) o número de sistemas que receberam uma avaliação 3; e (5) o número de sistemas que receberam uma avaliação 4. Por se tratar de uma população em idade laboral em que saúde muito precária é incompatível com as atividades cotidianas, foram utilizados para fins de análise apenas o número de sistemas/órgãos afetados e o escore total.

Em relação ao julgamento clínico da CIRS, duas enfermeiras (A.S. e J.C.), uma pesquisadora e outra especialista em enfermagem do trabalho foram treinadas para preenchimento da escala. A enfermeira AS, após um mês da aplicação, replicou a classificação para todos os indivíduos visando avaliar a confiabilidade intraobservador da escala. Na confiabilidade intraobservador, o coeficiente de correlação intraclasse (CCI) para número de sistemas afetados foi 0,97 (intervalo de 95\% de confiança - IC95\%: 096-0,97) e escore total foi 0,92 (IC95\%: 0,91-0,93), ambos tidos como concordância quase perfeita segundo Landis \& Koch 30 . O CCI para número de sistemas afetados intraobservador foi de 0,84 (IC95\%: 0,81-0,86) e para escore total o valor foi de 0,65 (IC95\%: 0,61-0,69) classificados como concordância quase perfeita e concordância substancial, respectivamente 30 . 
Estudo anterior mostrou que a confiabilidade do preenchimento da CIRS foi comparável, se preenchida por enfermeira ou por médicos, indicando que enfermeiras treinadas podem realizar o preenchimento da CIRS de maneira aceitável sem entrevista direta com o indivíduo que está sendo avaliado 31 .

Sexo, idade (contínua) e escolaridade (até Ensino Médio completo e Superior completo ou mais) foram as covariáveis utilizadas na análise.

\section{Análise estatística}

Foram realizados testes $t$ de Student e manova para comparar as médias das variáveis contínuas (escore total, número de sistemas afetados e número de morbidades autorrelatadas) e teste de qui-quadrado para a variável categórica (multimorbidade). Modelos de regressão de Poisson com variância robusta, geral e estratificado por sexo, foram utilizados para estimar razões de prevalência (RP) e respectivos IC95\% do uso de serviços de saúde, segundo as variáveis de exposição. Tendo em conta que o uso de serviços de saúde na população geral é marcadamente influenciado por sexo, idade e escolaridade $32,33,34$, optou-se por estratificar as análises por sexo e utilizar as variáveis idade e escolaridade como variáveis de ajuste.

De acordo com o que foi sugerido por Barros \& Hirakata 35 , foram usados modelos de regressão de Poisson com variância robusta para obter uma estimativa direta da RP com o IC corrigido. A escolha desse método de regressão se justifica pela alta frequência do uso de serviços de saúde na população de estudo. Sob tais circunstâncias, a OR (odds ratio) superestimaria a RP. Além disso, a regressão de Poisson usando uma estimativa robusta da variância tem a vantagem de corrigir o IC, baseada em estimadores-sanduíche. As análises foram desenvolvidas no programa estatístico Stata versão 12.1 (https://www.stata.com).

\section{Aspectos éticos}

Os protocolos do Estudo Pró-Saúde 1999 e 2001 foram submetidos e aprovados pelos comitês de ética em pesquisa do Instituto de Medicina Social (461/2001) e do Hospital Universitário Pedro Ernesto (224/1999) - Universidade do Estado do Rio de Janeiro.

\section{Resultados}

Foram investigados 733 indivíduos que haviam respondido à pergunta sobre utilização de serviços de saúde (Tabela 1). A maior parte da população de estudo foi composta por mulheres $(66,2 \%)$, e a média de idade de homens e mulheres foi de 40,4 e 42,2 anos, respectivamente. Em ambos os sexos, predominou o menor nível de escolaridade, porém se observou uma maior proporção de mulheres com nível superior. Mulheres relataram uso de serviços um pouco mais frequentemente $(59,4 \%)$ do que os homens $(51,2 \%)$ (Tabela 1$)$.

A Tabela 2 apresenta a distribuição das diferentes medidas de multimorbidade segundo algumas características dos participantes. Mulheres apresentaram em média valores mais altos para escore total, número de sistemas afetados e número de morbidades autorrelatadas. Cerca de $51 \%$ das mulheres foram classificadas como apresentando multimorbidade. As faixas etárias mais avançadas apresentaram maiores valores para todas as medidas multimorbidade. Indivíduos com escolaridade até Ensino Médio completo tiveram valores mais altos das medidas de multimorbidade em relação aos indivíduos com Ensino Superior, assim como aqueles que utilizaram serviços de saúde em comparação aos que não utilizaram serviço de saúde.

A Tabela 3 mostra as RP brutas e ajustadas de uso de serviços de saúde para as quatro medidas de multimorbidade considerando toda a população do estudo e de acordo com sexo.

Considerando toda a população, na análise bruta, a probabilidade de ter usado o serviço de saúde aumentou em $4 \%$ para a cada unidade acrescida do escore total de multimorbidade, em $5 \%$ para cada sistema adicional afetado e em $9 \%$ a cada morbidade acrescida. Apresentar multimorbidade ( 2 ou mais doenças) esteve associada a um aumento de $30 \%$ na probabilidade de ter utilizado o serviço de 
Tabela 1

Características da população de estudo. Estudo Pró-Saúde, Rio de Janeiro, Brasil, 1999-2001.

\begin{tabular}{|c|c|c|c|}
\hline Característica & $\begin{array}{l}\text { Total } \\
\text { n (\%) }\end{array}$ & $\begin{array}{c}\text { Homens } \\
\text { n (\%) }\end{array}$ & $\begin{array}{c}\text { Mulheres } \\
\text { n (\%) }\end{array}$ \\
\hline Total & $733(100,0)$ & $248(33,8)$ & $485(66,2)$ \\
\hline Idade [média (mínimo-máximo)] & $41,6(24-69)$ & $40,4(24-58)$ & $42,2(25-69)$ \\
\hline \multicolumn{4}{|l|}{ Escolaridade } \\
\hline Até Ensino Médio completo & $434(59,2)$ & $159(64,1)$ & $275(56,7)$ \\
\hline Ensino Superior completo & $299(40,8)$ & $89(35,9)$ & $210(43,3)$ \\
\hline \multicolumn{4}{|l|}{ Uso de serviços de saúde } \\
\hline Sim & $415(56,6)$ & $127(51,2)$ & $288(59,4)$ \\
\hline Não & $318(43,4)$ & $121(48,8)$ & $197(40,6)$ \\
\hline
\end{tabular}

Tabela 2

Medidas de multimorbidade segundo variáveis de estudo. Estudo Pró-Saúde, Rio de Janeiro, Brasil, $1999-2001$.

\begin{tabular}{|c|c|c|c|c|}
\hline \multirow[t]{2}{*}{ Variáveis } & Escore total & $\begin{array}{c}\text { Número de sistemas } \\
\text { afetados }\end{array}$ & $\begin{array}{c}\text { Número de morbidades } \\
\text { autorrelatadas }\end{array}$ & Multimorbidade \\
\hline & Média (mínimo-máximo) & Média (mínimo-máximo) & Média (mínimo-máximo) & $\%(n)$ \\
\hline \multicolumn{5}{|l|}{ Sexo } \\
\hline Masculino & $5,6(0-15) *$ & $3,2(0-7) * *$ & $1,2(0-7)$ ** & $34,7(86) * \star$ \\
\hline Feminino & $6,3(0-16)$ & $3,6(0-9)$ & $1,8(0-9)$ & $51,1(248)$ \\
\hline \multicolumn{5}{|l|}{ Faixa etária (anos) } \\
\hline $24-34$ & $4,6(0-10) * \star$ & $2,7(0-6) * \star$ & $0,8(0-3) * *$ & $19,2(30) * \star$ \\
\hline $35-44$ & $5,9(0-16)$ & $3,4(0-9)$ & $1,3(0-9)$ & $38,9(124)$ \\
\hline $45-54$ & $7,2(0-16)$ & $4,1(0-8)$ & $2,3(0-8)$ & $66,2(137)$ \\
\hline 55 e mais & $7,7(1-13)$ & $4,6(1-8)$ & $3,2(0-7)$ & $84,3(43)$ \\
\hline \multicolumn{5}{|l|}{ Escolaridade } \\
\hline Até Ensino Médio completo & $6,5(0-15) * \star$ & $3,7(0-9) * \star$ & $1,8(0-9) * *$ & $52,5(228) * \star$ \\
\hline Ensino Superior completo & $5,6(0-16)$ & $3,2(0-8)$ & $1,3(0-6)$ & $35,5(106)$ \\
\hline \multicolumn{5}{|l|}{ Uso de serviços de saúde } \\
\hline Sim & $6,4(0-15) * \star$ & $3,6(0-8)$ * & $1,8(0-9) * *$ & $52,1(216) * \star$ \\
\hline Não & $5,7(0-16)$ & $3,3(0-9)$ & $1,3(0-8)$ & $37,1(118)$ \\
\hline
\end{tabular}

* Valor de $\mathrm{p} \leq 0,01$;

** Valor de $p \leq 0,001$

saúde. Após ajuste progressivo por idade e escolaridade, constata-se uma paulatina redução na força da associação entre uso de serviços de saúde e todos os indicadores de multimorbidade avaliados, permanecendo no modelo final associação estatisticamente significativa com uso de serviços de saúde apenas para o número de morbidades e multimorbidade (Tabela 3).

Nos modelos estratificados por sexo, a chance de utilizar o serviço de saúde se apresentou mais fortemente associada à multimorbidade entre homens, com uma RP de uso de serviços de saúde aumentada em 14\% para cada morbidade acrescida. Apresentar multimorbidade (2 ou mais doenças) esteve associada a um aumento de $43 \%$ na chance de homens utilizarem serviços de saúde. Entre mulheres, encontrou-se fraca associação entre multimorbidade e uso de serviços de saúde; após ajuste por idade e escolaridade, nenhuma medida de multimorbidade foi associada com uso de serviços de saúde (Tabela 3). 
Tabela 3

Razões de prevalência (RP) bruta e ajustada e respectivos intervalos de 95\% de confiança (IC95\%) para uso de serviços de saúde segundo medidas de multimorbidade. Estudo Pró-Saúde, Rio de Janeiro, Brasil, 1999-2001.

\begin{tabular}{|c|c|c|c|}
\hline Índices de multimorbidade & $\begin{array}{c}\text { Modelo } 1 \\
\text { RP (IC95\%) }\end{array}$ & $\begin{array}{c}\text { Modelo } 2 \\
\text { RP (IC95\%) }\end{array}$ & $\begin{array}{c}\text { Modelo } 3 \\
\text { RP (IC95\%) }\end{array}$ \\
\hline \multicolumn{4}{|l|}{ Total } \\
\hline Escore total & $1,04(1,01-1,06)$ * & $1,02(1,00-1,04) * *$ & $1,02(0,99-1,04)$ \\
\hline Número de sistemas afetados & $1,05(1,01-1,09) * \star *$ & $1,03(0,99-1,07)$ & $1,02(0,98-1,06)$ \\
\hline Número de morbidades & $1,09(1,05-1,13)$ * & $1,05(1,01-1,10) * \star$ & $1,05(1,00-1,09) * *$ \\
\hline Multimorbidade & $1,30(1,14-1,47)$ * & $1,19(1,04-1,37) * \star *$ & $1,18(1,03-1,35) * *$ \\
\hline \multicolumn{4}{|l|}{ Homens } \\
\hline Escore total & $1,05(1,01-1,10) * \star \star$ & $1,03(0,99-1,08)$ & $1,03(0,99-1,08)$ \\
\hline Número de sistemas afetados & $1,09(1,02-1,18) * *$ & $1,06(0,98-1,14)$ & $1,06(0,98-1,15)$ \\
\hline Número de morbidades & $1,18(1,10-1,27)$ * & $1,14(1,05-1,23) * \star \star$ & $1,14(1,05-1,24) * \star \star$ \\
\hline Multimorbidade & $1,58(1,26-2,00)$ * & $1,43(1,11-1,84) * \star \star$ & $1,43(1,11-1,84) * * *$ \\
\hline \multicolumn{4}{|l|}{ Mulheres } \\
\hline Escore total & $1,03(1,00-1,05) * *$ & $1,02(0,99-1,04)$ & $1,01(0,99-1,04)$ \\
\hline Número de sistemas afetados & $1,03(0,98-1,08)$ & $1,01(0,97-1,06)$ & $1,01(0,96-1,05)$ \\
\hline Número de morbidades & $1,05(1,00-1,10) * *$ & $1,02(0,96-1,07)$ & $1,01(0,96-1,06)$ \\
\hline Multimorbidade & $1,16(1,00-1,35) * *$ & $1,08(0,91-1,27)$ & $1,04(0,88-1,22)$ \\
\hline
\end{tabular}

Nota: Modelo 1: análise bruta; Modelo 2: ajustado por idade; Modelo 3: ajustado por idade e escolaridade.

* Valor de $\mathrm{p} \leq 0,001$;

$\star \star$ Valor de $\mathrm{p} \leq 0,05$;

$\star \star \star$ Valor de $p \leq 0,01$.

\section{Discussão}

Neste estudo, avaliou-se a associação entre diferentes medidas de multimorbidade e o uso de serviços de saúde em uma população em idade laboral com restrição recente de atividades habituais por motivos de saúde. Foram incluídas pessoas que não utilizaram serviços de saúde, permitindo ampliar o escopo da literatura sobre o tema, comumente restrita a populações de usuários de tais serviços 22,34,36,37.

Neste estudo, o número de morbidades autorrelatadas esteve diretamente associado ao uso de serviços de saúde. $\mathrm{O}$ ajuste por idade resultou em redução da força da associação entre o uso de serviços de saúde e o número de morbidades autorrelatadas, provavelmente por estar diretamente relacionada ao aumento da morbidade e ao uso de serviços de saúde 7,34,38,39,40. Da mesma forma, o ajuste por escolaridade também reduziu a força da associação de interesse.

Um estudo avaliando o uso de serviços de saúde segundo posição socioeconômica (mensurada por meio da escolaridade), realizado na mesma população, encontrou proporções significativamente maiores de uso de serviços no menor nível de escolaridade e entre trabalhadores manuais, com gradiente dose-resposta 41 . Nesse estudo, indivíduos com menor escolaridade fizeram maior uso dos serviços de saúde quando em restrição de atividades habituais, mesmo após o ajuste por sexo, idade e demais marcadores de posição socioeconômica.

Outros estudos de base populacional que avaliaram a equidade no uso de serviços de saúde em que foi relatada restrição de atividades habituais nos últimos 15 dias, encontraram resultado inverso, com maior uso pelos mais ricos e de maior escolaridade 42,43. Entretanto, na população do presente estudo, possivelmente por se tratarem de funcionários públicos, indivíduos em situação de extrema desvantagem socioeconômica (e.g. desempregados) não foram incluídos, o que pode explicar em parte essa diferença ${ }^{41}$.

Além disso, no estudo de Pavão et al. 41, a ocupação foi o marcador de posição socioeconômica com o maior gradiente, sugerindo que diferenças nos padrões das relações de trabalho que possam 
ter influenciado o uso diferenciado de serviços nos diferentes estratos ocupacionais. A necessidade de apresentar atestado médico para justificar a ausência do trabalho poderia ocorrer especialmente para os funcionários que contam com menor flexibilidade em relação a suas chefias (trabalhadores de rotina manual e não manual) e pode ter ocasionado maior uso por serviços. Isso não ocorreria entre os indivíduos com maior flexibilidade em suas relações de trabalho. Ademais, a proximidade geográfica do hospital universitário potencialmente diminui barreiras de acesso ao serviço e esse comportamento tenderia a ser mais frequente. O que pode explicar em parte a redução da força de associação. Outro fator que justificaria essa redução é que baixa escolaridade está diretamente associada ao aumento das condições crônicas coexistindo em um mesmo indivíduo 7,44,45.

As análises ajustadas por idade e escolaridade, quando estratificadas por sexo, revelaram um padrão diferente na associação de interesse. Entre homens, a prevalência de uso do serviço de saúde foi $14 \%$ maior a cada morbidade acrescida. Para multimorbidade classificada de forma dicotômica, a RP do uso de serviços de saúde foi $43 \%$ maior entre os participantes com duas morbidades ou mais em relação àqueles com uma ou nenhuma morbidade. Em contraste, entre mulheres não houve associação estatisticamente significativa para qualquer medida de multimorbidade nos modelos ajustados por idade e escolaridade.

Ao contrário dos achados deste trabalho, Bähler et al. 46 encontraram que a presença de multimorbidade aumentou em cerca de 3,5 vezes o número de consultas tanto para homens quanto para mulheres. Interessante notar, contudo, que, quando ajustado por multimorbidade, o número de consultas entre homens aumentou com a idade, enquanto houve um decréscimo entre as mulheres, ainda que as mulheres tenham mais multimorbidade que os homens. Além disso, em indivíduos de 85 anos ou mais, houve diminuição significativa das consultas em mulheres, verificando-se aumento em homens da mesma idade 46.

Por outro lado, um estudo na Espanha encontrou que, entre os pacientes com multimorbidade, o número médio de visitas a serviços de saúde foi maior entre mulheres até 65 anos de idade, mas se tornou similar nos grupos etários mais avançados 47 . Em outro estudo, foi observado que apesar da maior frequência de multimorbidade entre mulheres, homens com multimorbidade eram mais atendidos em atenção primária do que mulheres 48 .

Estudos sobre utilização de serviços de saúde na população em geral relatam que mulheres fazem mais visitas aos centros de atenção primária do que os homens, o que pode ser atribuído à sua atitude mais proativa em relação à prevenção, a um pior estado de saúde ou ao fato de acompanharem familiares em suas visitas aos médicos 49.

No Brasil, mulheres utilizam mais os serviços de saúde mesmo quando controlando para o efeito da restrição das atividades por motivo de saúde, utilizam mais os serviços preventivos, assim como também possuem maiores taxas de internação, mesmo excluindo os partos 50,51. Além disso, as mulheres buscam mais serviços para realização de exames de rotina e prevenção, enquanto os homens procuram serviços de saúde dominantemente por motivo de doença 50 . O padrão de utilização de serviços de saúde de um determinado grupo populacional é predominantemente explicado por seu perfil de necessidades em saúde 52 , sendo assim, tal peculiaridade em termos do perfil de serviços buscado por homens e mulheres pode explicar, pelo menos em parte, as diferenças de gênero no perfil do uso de serviço de saúde encontradas no presente estudo.

As medidas produzidas pela CIRS não apresentaram associação estatisticamente significativa com o uso de serviços de saúde. A CIRS foi construída inicialmente para atender à necessidade de um instrumento curto, porém abrangente, válido e confiável, para avaliar a carga de morbidade em indivíduos 53; foi considerada como tendo boa validade de face, tendo sido adaptada e validada em outros contextos 29,31,54,55,56,57. A confiabilidade inter e intraobservador da CIRS foram avaliadas como satisfatórias tanto para seu preenchimento por meio de entrevistas quanto pela revisão de prontuários 31 .

A contagem de doença é mais utilizada em relação a medidas ponderadas como a CIRS pela facilidade de mensuração, não requerendo um julgamento individual, nem de pessoal treinado, além de ser mais viável no caso de inquéritos e em estudos baseados em registros eletrônicos ou administrativos. A maior parte dos estudos de multimorbidade em atenção primária se baseia em revisão de registros de saúde 58. A desvantagem de usar a CIRS em tais revisões é que requer mapeamento adicional de diagnósticos do sistema de classificação em que os registros de saúde foram codificados 59. 
No entanto, medidas que incluem uma avaliação subjetiva do estado funcional ou carga de doenças parecem ser preditores mais fortes de uma série de resultados de saúde do que aqueles que contam doenças sem ajuste para a sua gravidade ou impacto 60,61. Estudos que usaram a CIRS como medida para multimorbidade trabalharam com dados de prontuários médicos ou entrevistas diretamente com os indivíduos de estudo, permitindo obter informações mais detalhadas sobre a situação de saúde, facilitando o julgamento clínico na aplicação da escala 31,54,60. Em nosso estudo, a restrição a dados de autorrelato pode ter motivado erros não diferenciais de classificação dos indivíduos, anulando possíveis associações da multimorbidade medida pela CIRS com uso de serviços de saúde.

Como limitação adicional do estudo, deve-se destacar que a lista de morbidades autorrelatadas utilizadas para a classificação de contagem simples abordou apenas 12 diagnósticos, fato que pode ter reduzido as estimativas de multimorbidade entre os indivíduos avaliados. A padronização da forma de mensuração da multimorbidade ainda é uma agenda em aberto 58,62 . Nesse sentido, a construção de instrumentos que captem melhor o construto da multimorbidade para a gama de dados administrativos disponíveis é um dos desafios a serem enfrentados.

Outra limitação do estudo se deve ao fato de a pergunta sobre busca por serviços de saúde ter estado vinculada à pergunta sobre restrição de atividades habituais. Indivíduos que não tiveram interrupção de suas atividades, mas que podem ter procurado e utilizado serviços de saúde como forma de prevenção ou por razões de rotina podem não ter sido captados, subestimando então o uso de serviços de saúde dentre tal população.

Além disso, é preciso destacar que a opção por realizar análises estratificadas por sexo pode ter levado à redução do poder do estudo para detectar diferenças como estatisticamente significantes. Entretanto, mesmo reconhecendo esse problema, entende-se que a estratificação por sexo é uma necessidade nesse contexto, levando em consideração o amplo reconhecimento na literatura de que os padrões de uso de serviços de saúde são marcadamente diferentes no que concerne ao sexo. Por fim, utilizar busca como proxy pode superestimar o uso de serviços de saúde, pois indivíduos que buscaram serviços de saúde podem não ter conseguido atendimento.

\section{Conclusão}

Conhecer os padrões de uso de serviços de saúde segundo multimorbidade é relevante dada a sua alta prevalência, gravidade e impacto na qualidade de vida. A associação entre multimorbidade e uso de serviços de saúde variou de acordo com o sexo dos participantes, revelando-se mais forte entre homens.

Particularmente, as diferenças de gênero no uso de serviços de saúde em indivíduos com multimorbidade ainda tem sido pouco explorada e mais estudos são necessários para conhecer os padrões de uso.

Ademais, o impacto da multimorbidade no aumento do uso dos serviços de saúde requer a ruptura com a forma atual de prestação de cuidado organizado por meio de "programas verticais" centrados em uma única patologia. Além disso, é necessário garantir a coordenação do cuidado nos distintos níveis e complexidade, evitando, assim, tratamentos concorrentes ou replicação desnecessária de exames diagnósticos.

Por fim, compreender a relação do uso de serviços de saúde segundo multimorbidade no Brasil ainda é uma tarefa em aberto, frente a um cenário cada vez mais propenso ao aumento da prevalência de doenças crônicas coexistindo em um mesmo indivíduo e a uma limitada capacidade dos sistemas de saúde para gerenciar múltiplas morbidades. 


\section{Colaboradores}

A. S. S. Souza, E. Faerstein e G. L. Werneck contribuíram com a concepção do projeto, análise e interpretação dos dados, redação do artigo e aprovação final da versão a ser publicada.

\section{Informações adicionais}

ORCID: Ana Sara Semeão de Souza (0000-00024554-1551); Eduardo Faerstein (0000-0002-40274896); Guilherme Loureiro Werneck (0000-00031169-1436).

\section{Agradecimentos}

À Coordenação de Aperfeiçoamento de Pessoal de Nível Superior (Capes) pela concessão da bolsa de estudos do curso de mestrado.

\section{Referências}

1. World Health Organization. Global status report on noncommunicable diseases 2010. Description of the global burden of NCDs, their risk factors and determinants. Geneva: World Health Organization; 2011.

2. Lozano R, Naghavi M, Foreman K, Lim S, Shibuya K, Aboyans V, et al. Global and regional mortality from 235 causes of death for 20 age groups in 1990 and 2010: a systematic analysis for the Global Burden of Disease Study 2010. Lancet 2012; 380:2095-128.

3. Vasconcelos AMN, Gomes MMF. Transição demográfica: a experiência brasileira. Epidemiol Serv Saúde 2012; 21:539-48.

4. Schramm JMA, Oliveira AF, Leite IC, Valente JG, Gadelha ÂMJ, Portela MC, et al. Epidemiological transition and the study of burden of disease in Brazil. Ciênc Saúde Colet 2004; 9:897-908.

5. Rzewuska M, de Azevedo-Marques JM, Coxon D, Zanetti ML, Zanetti AC, Franco LJ, et al. Epidemiology of multimorbidity within the Brazilian adult general population: evidence from the 2013 National Health Survey (PNS 2013). PLoS One 2017; 12:e0171813.

6. Nunes BP, Chiavegatto Filho ADP, Pati S, Cruz Teixeira DS, Flores TR, Camargo-Figuera FA, et al. Contextual and individual inequalities of multimorbidity in Brazilian adults: a crosssectional national-based study. BMJ Open 2017; 7:e015885.

7. Nunes BP, Camargo-Figuera FA, Guttier M, de Oliveira PD, Munhoz TN, Matijasevich A, et al. Multimorbidity in adults from a southern Brazilian city: occurrence and patterns. Int J Public Health 2016; 61:1013-20.

8. Araujo MEA, Silva MT, Galvao TF, Nunes BP, Pereira MG. Prevalence and patterns of multimorbidity in Amazon Region of Brazil and associated determinants: a cross-sectional study. BMJ Open 2018; 8:e23398.

9. Carvalho JN, de Camargo Cancela M, de Souza DLB. Lifestyle factors and high body mass index are associated with different multimorbidity clusters in the Brazilian population. PLoS One 2018; 13:e0207649.

10. Costa CD, Flores TR, Wendt A, Neves RG, Tomasi E, Cesar JA, et al. Inequalities in multimorbidity among elderly: a population-based study in a city in Southern Brazil. Cad Saúde Pública 2018; 34:e00040718.

11. Jantsch AG, Alves RFS, Faerstein E. Educational inequality in Rio de Janeiro and its impact on multimorbidity: evidence from the ProSaude study. A cross-sectional analysis. São Paulo Med J 2018; 136:51-8.

12. Costa JSD, Victora CG. O que é "um problema de saúde pública”? Rev Bras Epidemiol 2006; 9:144-6.

13. Laranjeira CA. O contexto organizacional e a experiência de estresse: uma perspectiva integrativa. Rev Salud Pública 2009; 11:123-33. 
14. Johns G. Presenteeism in the workplace: a review and research agenda. J Organ Behav 2010; 31:519-42.

15. Rasmussen B, Sweeny K, Sheehan P. Economic costs of absenteeism, presenteeism and early retirement due to ill health: a focus on Brazil. Melbourne: Victoria Institute of Strategic Economic Studies; 2015.

16. Glynn LG, Valderas JM, Healy P, Burke E, Newell J, Gillespie P, et al. The prevalence of multimorbidity in primary care and its effect on health care utilization and cost. Fam Pract 2011; 28:516-23.

17. Lehnert T, Heider D, Leicht H, Heinrich S, Corrieri S, Luppa M, et al. Review: health care utilization and costs of elderly persons with multiple chronic conditions. Med Care Res Rev 2011; 68:387-420.

18. Starfield B. Challenges to primary care from co- and multi-morbidity. Prim Health Care Res Dev 2011; 12:1-2.

19. Violan C, Foguet-Boreu Q, Flores-Mateo G, Salisbury C, Blom J, Freitag M, et al. Prevalence, determinants and patterns of multimorbidity in primary care: a systematic review of observational studies. PLoS One 2014; 9:e102149.

20. Bambra C, Gibson M, Sowden A, Wright K, Whitehead M, Petticrew M. Tackling the wider social determinants of health and health inequalities: evidence from systematic reviews. J Epidemiol Community Health 2010; 64:28491.

21. Salisbury C, Johnson L, Purdy S, Valderas JM, Montgomery AA. Epidemiology and impact of multimorbidity in primary care: a retrospective cohort study. Br J Gen Pract 2011; 61:e1221.

22. van Oostrom SH, Picavet HS, de Bruin SR, Stirbu I, Korevaar JC, Schellevis FG, et al. Multimorbidity of chronic diseases and health care utilization in general practice. BMC Fam Pract 2014; 15:61.

23. Faerstein E, Chor D, Lopes CS, Werneck GL. The Pro-Saude Study: general characteristics and methodological aspects. Rev Bras Epidemiol 2005; 8:454-66.

24. Faerstein E, Lopes CS, Valente K, Solé Plá MA, Ferreira MB. Pré-testes de um questionário multidimensional autopreenchível: a experiência do Estudo Pró-Saúde UERJ. Physis (Rio J.) 1999; 9:117-30.

25. Instituto Brasileiro de Geografia e Estatística. Acesso e utilização de serviços de saúde. Brasil, 1998. Rio de Janeiro: Instituto Brasileiro de Geografia e Estatística; 2000.

26. Instituto Brasileiro de Geografia e Estatística. Acesso e utilização de serviços de saúde. Brasil, 2003. Rio de Janeiro: Instituto Brasileiro de Geografia e Estatística; 2005.

27. Instituto Brasileiro de Geografia e Estatística. Um panorama da saúde no Brasil: acesso e utilização dos serviços, condições de saúde e fatores de risco e proteção à saúde, 2008. Rio de Janeiro: Instituto Brasileiro de Geografia e Estatística; 2010.
28. Instituto Brasileiro de Geografia e Estatística. Pesquisa Nacional de Saúde, 2013: acesso e utilização dos serviços de saúde, acidentes e violências. Brasil, grandes regiões e unidades da federação. Rio de Janeiro: Instituto Brasileiro de Geografia e Estatística; 2015.

29. Miller MD, Paradis CF, Houck PR, Mazumdar S, Stack JA, Rifai AH, et al. Rating chronic medical illness burden in geropsychiatric practice and research: application of the Cumulative Illness Rating Scale. Psychiatry Res 1992; 41:237-48.

30. Landis JR, Koch GG. The measurement of observer agreement for categorical data. Biometrics 1977; 33:159-74.

31. Hudon C, Fortin M, Vanasse A. Cumulative Illness Rating Scale was a reliable and valid index in a family practice context. J Clin Epidemiol 2005; 58:603-8.

32. Dias-da-Costa JS, Gigante DP, Horta BL, Barros FC, Victora CG. Utilização de serviços de saúde por adultos da coorte de nascimentos de 1982 a 2004-5, Pelotas, RS. Rev Saúde Pública 2008; 42 Suppl 2:51-9.

33. Dias-da-Costa JS, Facchini LA. Use of outpatient services in an urban area of Southern Brazil: place and frequency. Rev Saúde Pública 1997; 31:360-9.

34. Fernandes LC, Bertoldi AD, Barros AJ. Health service use in a population covered by the Estratégia de Saúde da Família (Family Health Strategy). Rev Saúde Pública 2009; 43:595603.

35. Barros AJ, Hirakata VN. Alternatives for logistic regression in cross-sectional studies: an empirical comparison of models that directly estimate the prevalence ratio. BMC Med Res Methodol 2003; 3:21.

36. van den Bussche H, Schon G, Kolonko T, Hansen H, Wegscheider K, Glaeske G, et al. Patterns of ambulatory medical care utilization in elderly patients with special reference to chronic diseases and multimorbidity: results from a claims data based observational study in Germany. BMC Geriatr 2011;11:54.

37. Ranstad K, Midlov P, Halling A. Importance of healthcare utilization and multimorbidity level in choosing a primary care provider in Sweden. Scand J Prim Health Care 2014; 32:99105.

38. Walker AE. Multiple chronic diseases and quality of life: patterns emerging from a large national sample, Australia. Chronic Illn 2007; 3:202-18.

39. Lee JT, Hamid F, Pati S, Atun R, Millett C. Impact of noncommunicable disease multimorbidity on healthcare utilisation and out-ofpocket expenditures in middle-income countries: cross sectional analysis. PLoS One 2015; 10:e0127199.

40. Pati S, Agrawal S, Swain S, Lee JT, Vellakkal $\mathrm{S}$, Hussain MA, et al. Non communicable disease multimorbidity and associated health care utilization and expenditures in India: crosssectional study. BMC Health Serv Res 2014; 14:451. 
41. Pavão ALB, Coeli CM, Lopes CS, Faerstein E, Werneck GL, Chor D. Uso de serviços de saúde segundo posição socioeconômica em trabalhadores de uma universidade pública. Rev Saúde Pública 2012; 46:98-103.

42. Travassos C, Oliveira EXG, Viacava F. Desigualdades geográficas e sociais no acesso aos serviços de saúde no Brasil: 1998 e 2003. Ciênc Saúde Colet 2006; 11:975-86.

43. Chiavegatto Filho AD, Wang YP, Malik AM, Takaoka J, Viana MC, Andrade LH. Determinants of the use of health care services: multilevel analysis in the Metropolitan Region of Sao Paulo. Rev Saúde Pública 2015; 49:15.

44. Yates WR, Mitchell J, John Rush A, Trivedi M, Wisniewski SR, Warden D, et al. Clinical features of depression in outpatients with and without co-occurring general medical conditions in STAR*D: confirmatory analysis. Prim Care Companion J Clin Psychiatry 2007; 9:715.

45. Heyworth IT, Hazell ML, Linehan MF, Frank TL. How do common chronic conditions affect health-related quality of life? Br J Gen Pract 2009; 59:e353-8.

46. Bähler C, Huber CA, Brüngger B, Reich O. Multimorbidity, health care utilization and costs in an elderly community-dwelling population: a claims data based observational study. BMC Health Serv Res 2015; 15:23.

47. Violan C, Foguet-Boreu Q, Roso-Llorach A, Rodriguez-Blanco T, Pons-Vigues M, PujolRibera E, et al. Burden of multimorbidity, socioeconomic status and use of health services across stages of life in urban areas: a cross-sectional study. BMC Public Health 2014; 14:530.

48. Fortin M, Hudon C, Haggerty J, van den Akker M, Almirall J. Prevalence estimates of multimorbidity: a comparative study of two sources. BMC Health Serv Res 2010; 10:111.

49. Borrell C, Palencia L, Rodriguez-Sanz M, Malmusi D, Bartoll X, Puigpinos R. Trends in social inequalities in health in Catalonia, Spain. Med Clin (Barc) 2011; 137 Suppl 2:60-5.

50. Pinheiro RS, Viacava F, Travassos C, Brito AS. Gender, morbidity, access and utilization of health services in Brazil. Ciênc Saúde Colet 2002; 7:687-707.

51. Travassos C, Viacava F, Pinheiro R, Brito A. Utilization of health care services in Brazil: gender, family characteristics, and social status. Rev Panam Salud Pública 2002; 11:365-73.

52. Hulka BS, Wheat JR. Patterns of utilization. The patient perspective. Med Care 1985; 23:438-60.
53. Linn BS, Linn MW, Gurel L. Cumulative illness rating scale. J Am Geriatr Soc 1968; 16:622-6.

54. Fortin M, Steenbakkers K, Hudon C, Poitras ME, Almirall J, van den Akker M. The electronic Cumulative Illness Rating Scale: a reliable and valid tool to assess multi-morbidity in primary care. J Eval Clin Pract 2011; 17:108993.

55. Parmelee PA, Thuras PD, Katz IR, Lawton MP. Validation of the Cumulative Illness Rating Scale in a geriatric residential population. J Am Geriatr Soc 1995; 43:130-7.

56. Extermann M, Overcash J, Lyman GH, Parr J, Balducci L. Comorbidity and functional status are independent in older cancer patients. J Clin Oncol 1998; 16:1582-7.

57. Castillo C, Bulbena A, Serras E, Torrens M, Lopez-Colomes JL, Martinez MA, et al. Medical assessment in drug addicts: reliability and validity of the Cumulative Illness Rating Scale (Substance Abuse version). Eur Addict Res 2004; 10:112-7.

58. Fortin M, Stewart M, Poitras ME, Almirall J, Maddocks H. A systematic review of prevalence studies on multimorbidity: toward a more uniform methodology. Ann Fam Med 2012; 10:142-51.

59. Harrison C, Britt H, Miller G, Henderson J. Examining different measures of multimorbidity, using a large prospective cross-sectional study in Australian general practice. BMJ Open 2014; 4:e004694.

60. Fortin M, Hudon C, Dubois MF, Almirall J, Lapointe L, Soubhi H. Comparative assessment of three different indices of multimorbidity for studies on health-related quality of life. Health Qual Life Outcomes 2005; 3:74.

61. Bayliss EA, Ellis JL, Steiner JF. Seniors' selfreported multimorbidity captured biopsychosocial factors not incorporated into two other data-based morbidity measures. J Clin Epidemiol 2009; 62:550-7.e1.

62. Diederichs C, Berger K, Bartels DB. The measurement of multiple chronic diseases: a systematic review on existing multimorbidity indices. J Gerontol A Biol Sci Med Sci 2011; 66:301-11. 


\section{Abstract}

The study aimed to assess the use of health services and the association with different measures of multimorbidity. This was a cross-sectional study nested in the Pró-Saúde Study, a longitudinal study of municipal technical and administrative employees in Rio de Janeiro, Brazil. Data were analyzed from phase 2 (2001-2002), and the study population consisted of 733 individuals who reported restrictions on habitual activities due to health problems in the 15 days prior to the data collection. The search for a health service (outcome variable) was used as the proxy for use of health services. Multimorbidity was assessed by simple count and the Cumulative Illness Rating Scale, generating four exposure variables: number of self-reported diseases, multimorbidity (2 or more diseases), and total score and number of systems affected. The analyses stratified by sex used Poisson regression models with robust variance, adjusted by age and schooling. Women showed higher mean values than men for all the measures, with $51 \%$ classified as having multimorbidity. In men, multimorbidity increased by $43 \%$ (95\% CI: 1.11-1.84) the probability of using health services, while there was no statistically significant association in women. For men, each additional disease increased the probability of use of a health service by 14\% (95\%CI: 1.05-1.24). There were evident differences in the use of health services and multimorbidity according to sex. Explaining these patterns becomes relevant for the provision of efficient, coordinated, and safe care for persons with multimorbidity.

Multiple Morbidity; Comorbidity; Health Services Accessibility; Chronic Disease; Health Systems

\section{Resumen}

El objetivo del estudio fue evaluar el uso de servicios de salud y su asociación con diferentes medidas de multimorbilidad. Se trata de un estudio transversal, anidado en una investigación longitudinal de funcionarios técnico-administrativos, en el municipio de Rio de Janeiro, Brasil: Estudio Pro-Salud. Se analizaron los datos recogidos en la fase 2(2001-2002), estando la población de estudio compuesta por 733 individuos que relataron restricción de actividades habituales por problemas de salud, durante los 15 días anteriores a la recogida de datos. Búsqueda de un servicio de salud (variable de desenlace) se utilizó como proxy para el uso de servicios de salud. La multimorbilidad se evaluó mediante cómputo simple y una escala acumulativa (Cumulative Illness Rating Scale), generando cuatro variables de exposición: número de morbilidades autoinformadas, multimorbilidad (2 o más morbilidades), puntuación total y número de sistemas afectados. En los análisis estratificados por sexo se utilizaron modelos de regresión de Poisson con variancia robusta ajustados por edad $y$ escolaridad. Las mujeres presentaron de media valores más altos para todas las medidas, siendo que un $51 \%$ fueron clasificadas con multimorbilidad. Tener multimorbilidad aumentó en un $43 \%$ (IC95\%: 1,11-1,84) la probabilidad de utilizar los servicios de salud en hombres, mientras que para las mujeres no hubo asociación estadísticamente significativa. Para los hombres, con cada morbilidad adicional, la probabilidad de utilizar un servicio de salud aumentó en un 14\% (IC95\%: 1,05-1,24). Las diferencias en el uso de servicios de salud y multimorbilidad según sexo son evidentes. Conocer estos patrones es relevante para la prestación de un cuidado eficiente, coordinado y seguro para personas con multimorbilidad.

Múltiples Morbilidades; Comorbilidad;

Accesibilidad a los Servicios de Salud;

Enfermedad Crónica; Sistemas de Salud
Recebido em 07/Ago/2018

Versão final reapresentada em 29/Abr/2019

Aprovado em 16/Mai/2019 